\title{
Mapping Regional Ecosystem Functional Types Based on Sentinel-2 Satellite Imagery
}

\author{
Rong Liu, Fang Huang*, and Yue Ren
}

Key Laboratory of Geographical Processes and Ecological Security in Changbai Mountains, Ministry of Education, School of Geographical Sciences, Northeast Normal University, Renmin Street No.5268, Changchun 130024, China

\begin{abstract}
Ecosystem functional types (EFTs) are the patches of land surface showing similar in carbon dynamics. EFTs are not defined by the structure and composition of vegetation and represent the spatial heterogeneity of ecosystem functions. Identifying EFTs based on low-resolution satellite remote sensing data cannot satisfy the needs of fine-scale characterization of regional ecosystem functional patterns. Here, taking Zhenlai County, Northeast China as an example, the heterogeneity in ecosystem functions was characterized by identifying EFTs from Sentinel-2 time series data using ISODATA algorithm. Ecosystem functional attributes derived from dynamics of the normalized difference vegetation index (NDVI), the fraction of absorbed photosynthetically active radiation (FAPAR), and canopy water content (CWC) in the growing season were calculated. The correspondence analysis (CA) method was used to reveal relationships between the EFTs and land cover types. Our results showed that the nine selected remotely sensed variables indicating carbon and water flux of the regional ecosystems could be adopted in ecosystem functions classification. The obtained EFTs based on Sentinel-2 images reflected the internal structure of carbon balance well and the distribution pattern of ecosystem functional diversity a fine scale. This study helps to understand the functional heterogeneity pattern of temperate terrestrial ecosystems.
\end{abstract}

\section{Introduction}

Satellite remote sensing technology provides an effective means for terrestrial ecosystem assessment and monitoring at the regional to global scale. The term of ecosystem functional types (EFTs) was first proposed by Smith T.M. et al.[1] in 1997. Ecosystem functional attributes can be classified into functional units according to certain rules, which describe land surface areas with similar carbon dynamics independent of vegetation structure and composition[2]. As ecosystem groupings based on similar ecosystem functions, EFTs capture the spatial heterogeneity of ecosystem functions and regional biodiversity to a certain extent[3]. Satellite-derived multiple functioning properties can greatly contribute to the characterization of ecosystem heterogeneity and responses to environmental change, both in time and in space. For example, Wang et al.[4] used five indices derived from moderate resolution imaging spectroradiometer (MODIS) data products, namely, albedo, land surface water index, land surface temperature, Enhanced Vegetation Index, and net photosynthesis intensity and their derived variables, to identify ecosystem functional types. EFTs have been used to characterize the spatial patterns of ecosystem functioning at the global and regional scales[2-3,5-6]. However, previous studies have been conducted with low spatial resolution satellite data and cannot reveal ecosystem function heterogeneity at finer scales. Precise identification and analysis of EFTs using time series remote sensing data with higher spatial resolution is needed.

Sentinel-2 satellite offers global images with fine spatial resolutions, high revisit frequency and multispectral information. In this paper, taking Zhenlai County, Northeast China as an example, ecosystem functional types at a fine scale was characterized from Sentinel-2 time series data. The main objectives are to (1) select a series of parameters that can reflect the ecosystem functioning based on Sentinel-2 time series data; (2) identify EFTs in the Zhenlai County using ISODATA algorithm and perform the corresponding analysis of EFTs and ecosystem structure types. The study not only helps the exploration of more effective of revealing the spatial heterogeneity of ecosystem functions, but provides important reference for the protection of regional ecosystem diversity.

\section{Data and methods}

\subsection{Study area}

Zhenlai County is located between $122^{\circ} 47^{\prime}-124^{\circ} 04^{\prime}$ $\mathrm{E}$ and $45^{\circ} 28^{\prime}-46^{\circ} 18^{\prime} \mathrm{N}$ in the northwest of Jilin Province, China, with the total land area of $4737 \mathrm{~km}^{2}$. The county exhibits diversity with respect to land cover. It belongs to the mid-temperate continental monsoon climate. The annual average temperature is $4.9^{\circ} \mathrm{C}$ and the

\footnotetext{
* Corresponding author: huangf835@nenu.edu.cn
} 
annual average precipitation is $402 \mathrm{~mm}$. Also, there was a normal climate condition in 2018. Except for high tableland in the northwest, most of areas is a low-lying flood plain and sand dunes. The major soil types include chernozem, alluvium soil, alkali soil and meadow soil. Wind and sand, frequent drought, flood and land salinization are the main limiting factors in local agricultural production[7].

\subsection{Data and processing}

\subsubsection{Sentinel-2 Images}

Six cloud-free L1C-level Sentinel-2 images in May 7, June 1, July 31, August 10, September 9, and October 4, 2018 were acquired from the Sentinel Scientific Date Centre(ESA) (https://scihub.copernicus. eu/dhus/\#/home). The downloaded L1C-level top-of-the atmosphere (TOA) reflectance data were converted into L2A-level surface reflectance data by the ESA's Sen2Cor plug-in, which is in SNAP toolbox, version 02.08.00. Then, the adjacent images with the same acquisition date were then stitched together in ENVI5.3.

\subsubsection{Land Cover Data}

Land cover types data (FROM-GLC10) was downloaded from the Finer Resolution Observation and Monitoring of Global Land Cover (FROM-GLC) project at http://data. ess.tsinghua.edu.cn/. In this study, the land cover type map was converted to the same projection as the Sentinel2 data and was extracted the subset for the study area in ArcGIS10.5.

\subsection{Calculation of ecosystem functional classification index}

As one of the most commonly used vegetation index, NDVI can be used as a proxy for describing carbon gains. FAPAR has directly linkage to photosynthesis and therefore expresses the energy-absorption capacity of vegetation canopy[8]. Vegetation water content is a sensitive indicator of the strength of plant metabolism (e.g. photosynthesis, respiration, and transpiration)[9]. Therefore, we selected NDVI, FAPAR, and canopy water content (CWC) as indices for ecosystem function classification, which represent the functional attributes of ecosystem carbon gains, photosynthesis and water flux, respectively. NDVI has been used in the identification of EFTs[10]. The calculation formula is listed as follows:

$$
\mathrm{NDVI}=\frac{\rho_{\mathrm{NIR}}-\rho_{\mathrm{R}}}{\rho_{\mathrm{NIR}}+\rho_{\mathrm{R}}}
$$

where $\rho_{\mathrm{NIR}}$ and $\rho_{\mathrm{R}}$ are the near-infrared reflectance and the red band reflectance corresponding to band $8 \mathrm{~A}$ (848-881nm) and band 4(646-684nm) of Sentinel-2 data, respectively. Values of FAPAR and CWC for the whole Zhenlai County scene were extracted by the biophysical module in SNAP6.0 software.
Ecosystem functional attributes derived from NDVI, FAPAR, and CWC time series, namely, the mean value, maximum value and range (i.e. maximum-minimum values) in the growing season (May to October) of 2018 were calculated. The formulas are as follows:

$$
\begin{gathered}
y_{\text {meani }}=\frac{\sum_{j=5}^{I 0} x_{i j}}{6} \\
y_{\text {maxi }}=\max \left(x_{i 5}, x_{i 6}, x_{i 7}, x_{i 8}, x_{i 9}, x_{i 10}\right) \\
y_{\text {rangei }}=y_{\text {maxi }}-\min \left(x_{i 5}, x_{i 6}, x_{i 7}, x_{i 8}, x_{i 9}, x_{i 10}\right)
\end{gathered}
$$

where $y_{\text {meani }}$ is the average value over the growing season, corresponding to a specific index at pixel $i, i \in[1$, $n], j \in[1,6]$ represents the months in the growing season; $y_{\text {maxi }}$ represents the maximum value corresponding to a specific indicator at pixel $i$; and $y_{\text {rangei }}$ is the difference between the maximum value and the minimum value of a specific indicator at pixel $i$ in the year. Hence, nine ecosystem functional variable images were obtained to identify the EFTs for Zhenlai County.

\subsection{Ecosystem functional types identification and analysis}

We classified all pixels within the study area using the Iterative Self-Organizing Data Analysis (ISODATA) method based on the nine ecosystem functional variables. ISODATA is an unsupervised iterative classification technique that uses minimum distances to assign an individual pixel to a cluster. A new class mean is defined in each iteration based on the location of the pixel included in the cluster of the data space defined by the nine variables. We obtained eight classes within which the pixels had similar ecosystem functioning dynamics. We defined these classes as ecosystem functional types (EFTs).

The correspondence analysis (CA) is a method of data visualization and its results clarified the correlation among variables and the differences or similarities between classes of the same variable[2]. CA was used to associate the EFTs with land cover types (i.e. structural descriptions of ecosystem) in this study.

\section{Results and discussion}

\subsection{Analysis of regional ecosystem functional types in Zhenlai County, Northeast China}

From regional to global scales, EFTs approach was an alternative to quantify ecological functional diversity based on attributes related to processes. Here, the ecosystem functional classification was performed based on NDVI, FAPAR and CWC attributes derived from Sentinel-2 images. As shown as Table 1, the mean value of NDVI-Mean was 0.439 and the mean value of NDVIRange was 0.603 , indicating NDVI in vegetation areas had greater seasonal variations. The FAPAR variables mainly represented the changes in photosynthesis and carbon budget. The FAPAR-Mean varied from 0 to 0.8 , with an average value of 0.295 . Water content in the vegetation canopy is one of the main factors controlling 
vegetation photosynthesis, respiration and biomass. The CWC-Mean values varied from 0 to $0.269 \mu \mathrm{g} / \mathrm{cm}^{2}$. The low values indicated that the canopy water content in the growing season of 2018 was insufficient.

Table 1. The statistics of ecosystem functional classification indexes.

\begin{tabular}{cccc}
\hline Ecosystem functional index & Min & Max & Mean \\
\hline NDVI-Mean & -0.705 & 0.897 & 0.439 \\
NDVI-Max & -0.571 & 0.999 & 0.731 \\
NDVI-range & 0.004 & 1.973 & 0.603 \\
FAPAR-Mean & 0 & 0.8 & 0.295 \\
FAPAR-Max & 0 & 0.94 & 0.627 \\
FAPAR-range & 0 & 1.868 & 0.571 \\
CWC-Mean & 0 & 0.269 & 0.026 \\
CWC-Max & 0 & 0.297 & 0.059 \\
CWC-range & 0 & 0.425 & 0.053 \\
\hline
\end{tabular}

In the ISODATA analyses, we set the number of categories to 5 to 9 and the number of iterations to 50 . Nine initial functional groups were obtained, which were grouped into eight clusters, that is, the Ecosystem Functional Types. Fig.1. showed the EFTs map of Zhenlai County with water bodies removed (white areas). In addition, the percentage of each class areas of EFTs in the Zhenlai County were also shown. Among them, EFT4 and EFT5 were similar in area proportion, implying a relatively high heterogeneity of ecological function pattern in the study area. It can be seen that nearly half of the county was occupied by three ecosystem functional types, namely EFT2 (12.2\% accounting for the total land), EFT6 (15.1\%) and EFT7 (21.5\%), respectively.

The distribution of EFT1 was spatially located in the central and northwest part of Zhenlai County, with a small amount in the east. The values of the three variables NDVI, FAPAR and CWC were lower than other EFTs, which indicated that EFT1 may correspond to impervious surface and bareland. EFT2 mainly distributed in the eastern riverside area of Zhenlai County and discretely in the middle, similar to the areas covered by shrubland. The indicators of EFT2 were only higher than EFT1 but the value of CWC-Range is higher than EFT3.Thus, we inferred that EFT2 reflected functioning characteristics of shrubland with low vegetation cover, photosynthetic capacity but larger canopy water availability. EFT3 was overlapped with mixed cropland-forest areas and mixed cropland-grass areas. EFT3 was relatively continuous and mainly concentrated in the southeast and northwest. The NDVI-mean and FAPAR-mean of EFT3 are higher than EFT1 and EFT2, but the CWC-Range value is lower than EFT2. Therefore, EFT3 might correspond to the croplandforest mixed zone and cropland-grass mixed zone with medium coverage and photosynthetic capacity, and small changes in canopy water content.

EFT4 was mainly distributed in western parts of Zhenlai County. It was mainly covered by grassland and wetland, with values of NDVI and FAPAR at a medium level, indicating that EFT4 may correspond to grassland and wetland with low vegetation coverage and productivity. The distribution of EFT5 was scattered but showed a certain degree of aggregation, locating in forest and near-water-bodies cropland areas. Thus, we inferred that EFT5 might correspond to the cropland and sparse forest with medium coverage, canopy water content and slightly higher than EFT4. EFT6 was mainly distributed in the northwest parts of Zhenlai County and consistent with the spatial distribution of cropland, which was adjacent to grassland. It might correspond to cropland and mixed cropland-grass areas with relatively high vegetation coverage and its photosynthetic capacity higher than EFT5 but lower than EFT7.

The area proportion of EFT7 was highest, overlapped with almost all croplands in the county. The values of the three variables of NDVI, FAPAR and CWC of EFT7 were higher than other EFTs and only lower than those in EFT8, which indicated that EFT7 might correspond to cropland with a high coverage, strong photosynthetic capacity and good water condition of vegetation canopy. It also showed that the overall farmland in Zhenlai County showed a relatively good functional status. EFT8 was mainly concentrated in the southwest and east of the study area. The values of NDVI-Max and NDVI-Mean were high, probably associated with its distribution in low-lying alluvial plains along the river. Thus, we inferred that EFT8 might correspond to cropland with high productivity.

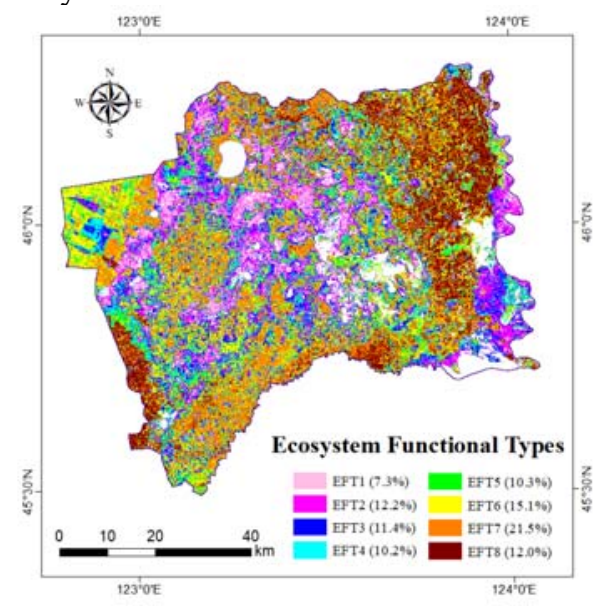

Fig. 1. Ecosystem functional types (EFTs) in Zhenlai County.

Table 2. illustrates the ecosystem functional classification index changes among different EFTs in Zhenlai County. Most of the EFTs had high vegetation coverage and productivity. The values of FAPAR-Max of EFT5, EFT6, EFT7and EFT8 were all greater than 0.6.

Table 2. Mean value of ecosystem functional classification indexes of each EFT in the study area.

\begin{tabular}{ccccccccc}
\hline Ecosystem functional index & EFT1 & EFT2 & EFT3 & EFT4 & EFT5 & EFT6 & EFT7 & EFT8 \\
\hline NDVI-Mean & 0.146 & 0.231 & 0.363 & 0.460 & 0.505 & 0.528 & 0.545 & 0.534 \\
NDVI-Max & 0.255 & 0.443 & 0.618 & 0.727 & 0.797 & 0.853 & 0.894 & 0.929 \\
NDVI-range & 0.213 & 0.414 & 0.501 & 0.536 & 0.602 & 0.681 & 0.742 & 0.837
\end{tabular}




\begin{tabular}{ccccccccc} 
FAPAR-Mean & 0.071 & 0.138 & 0.216 & 0.280 & 0.321 & 0.346 & 0.383 & 0.440 \\
FAPAR-Max & 0.179 & 0.323 & 0.462 & 0.569 & 0.657 & 0.748 & 0.830 & 0.881 \\
FAPAR-range & 0.181 & 0.299 & 0.397 & 0.479 & 0.574 & 0.692 & 0.780 & 0.801 \\
CWC-Mean & 0.009 & 0.017 & 0.017 & 0.019 & 0.022 & 0.025 & 0.031 & 0.052 \\
CWC-Max & 0.022 & 0.031 & 0.038 & 0.041 & 0.049 & 0.058 & 0.074 & 0.119 \\
CWC-range & 0.021 & 0.033 & 0.032 & 0.034 & 0.041 & 0.051 & 0.067 & 0.112 \\
\hline
\end{tabular}

It showed that most of ecosystem functional types in the study area exhibited good photosynthetic capacity. The value of CWC-Mean was low, most of EFTs in Zhenlai County had relatively low ecosystem water availability associated with the characteristics of the semi-arid area.

\subsection{Corresponding Relationship Between EFTs and Land Cover Types}

Fig. 2 depicted the correspondence of EFTs and land cover classification by means of a bi-dimensional plot in Zhenlai County. The comprehensive classification of EFTs and structure types explained $98 \%$ of the total of related frequency data on two dimensions $(73.9 \%$ and $24.1 \%$ for Dimension 1 and 2, respectively), which was a measure of variance of dispersion in the data. Among them, EFT1 was much associated with Impervious surface and EFT6 was the closest to cropland. Cropland was related to most EFTs, especially EFT6, EFT7, and EFT8, indicating the highly functional heterogeneity of this land cover type.

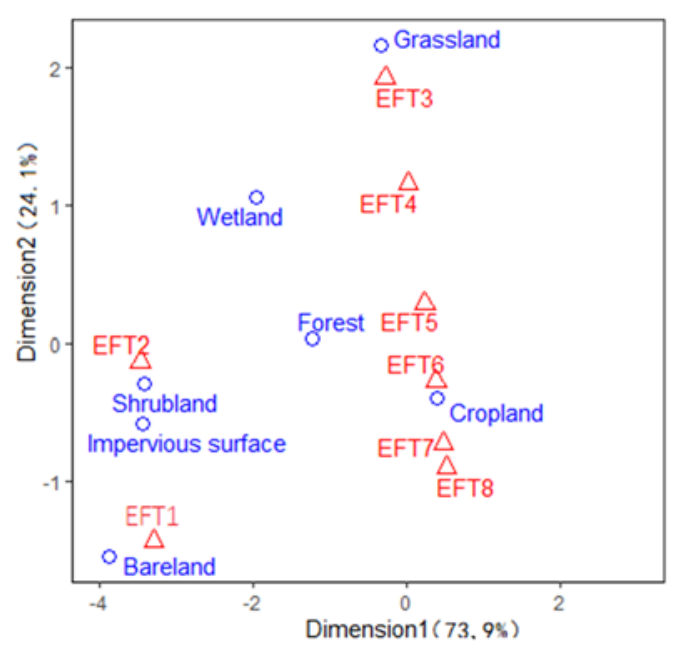

Fig. 2. Relationship between ecosystem functional types (EFTs) and land cover types by correspondence analysis.

\section{Conclusion}

The characterization of functional diversity at the ecosystem level using satellite remote sensing will guide conservation actions. Ecosystem functional types (EFTs), defined as areas exhibiting similar response to environmental conditions and similar ecosystem processes, are necessary to describe ecosystem status. Sentinel-2 data with $20 \mathrm{~m}$ spatial resolution used in this present study allowed an increase in the level of detail in the identification of EFTs, making the characterization of county-level EFTs meaningful. We used new satellitebased metrics or attributes (i.e. photosynthesis and water content of canopy). These indicators may expand the traditional definition of EFT (only based on the normalized vegetation index as an indicator of biomass) to include ecosystem vegetation photosynthesis and water balance, making it more comprehensive for identifying regional functional patterns of ecosystems and feedback on climate. Under the combined influence of natural and human factors, a novel and unique pattern of water, energy, and carbon balance developed in Zhenlai County.

\section{Acknowledgement}

This study was supported by the National Natural Science Foundation of China (Grant No. 41571405 and 41630749).

\section{References}

1. Smith, T.M.; Smith, T.M.; Shugart, H.H.; Woodward, F.I. Cambridge University Press, Vol. 1(1997).

2. Alcaraz, D.; Paruelo, J.; Cabello, J. Global Ecology and Biogeography, 15, 200-212(2006).

3. Paruelo, J.M.; Jobbágy, E.G.; Sala, O.E. Ecosystems, 4, 683-698 (2001).

4. Wang, Y.; Huang, F. Journal of Applied Remote Sensing . 9, 096096(2015).

5. Ivits, E.; Cherlet, M.; Horion, S.; Fensholt, R. Remote Sensing, 5, 3305-3330(2013).

6. Lara, B.; Gandini, M.; Gantes, P.; Matteucci, S.D. Ecological Informatics, 43, 65-72(2018).

7. Bao, C.H; Xu L.S, Wu, Y.J. Resources Science. 29, 92-96(2007).

8. Fensholt, R.; Sandholt, I.; Rasmussen, M.S.; Stisen, S.; Diouf, A. Remote Sensing of Environment, 105, 173-188(2006).

9. Ding, C.; Liu, X.; Huang, F.; Li, Y.; Zou, X. Onset of drying and dormancy in relation to water dynamics of semi-arid grasslands from MODIS NDWI. Agricultural and Forest Meteorology, 234, 2230(2017).

10. Alcaraz-Segura, D.; Paruelo, J.; Epstein, H.; Cabello, J. Remote Sensing, 5, 127-154( 2013). 